\title{
Diversity and function of bacterial microbiota in the mosquito holobiont
}

\author{
Guillaume Minard, Patrick Mavingui ${ }^{*}$ and Claire Valiente Moro*
}

\begin{abstract}
Mosquitoes (Diptera: Culicidae) have been shown to host diverse bacterial communities that vary depending on the sex of the mosquito, the developmental stage, and ecological factors. Some studies have suggested a potential role of microbiota in the nutritional, developmental and reproductive biology of mosquitoes. Here, we present a review of the diversity and functions of mosquito-associated bacteria across multiple variation factors, emphasizing recent findings. Mosquito microbiota is considered in the context of possible extended phenotypes conferred on the insect hosts that allow niche diversification and rapid adaptive evolution in other insects. These kinds of observations have prompted the recent development of new mosquito control methods based on the use of symbiotically-modified mosquitoes to interfere with pathogen transmission or reduce the host life span and reproduction. New opportunities for exploiting bacterial function for vector control are highlighted.
\end{abstract}

Keywords: Mosquito, Microbiome, Symbiont, Extended phenotype, Bacterial community

\section{Introduction}

Sustained relationships between prokaryotes and eukaryotes are known to be an important factor in the evolution and speciation of the interacting partners [1]. The classic example of this evolutionary process is the mitochondrion, an organelle essential for cell metabolism in eukaryotes that derives from a bacterial ancestor [2]. Another example is summed up in the coral probiotic hypothesis proposed by Reshef et al. (2006) which posits that corals can adapt to their environment by changing their symbiotic bacteria [3]. The symbiotic relationships between microbiota, whether algae, archaebacteria, eubacteria, protozoa or viruses, and their invertebrate host were shown to contribute to the acquisition of resistance to pathogens or tolerance to abiotic stresses [3,4]. Rosenberg et al. (2007) recently proposed the hologenome theory to explain such interactions between higher organisms and microbiota [5]. The hologenome theory is based on the concept that higher organisms are not dissociable from their microbial partners, and so together form a unit of selection in which genes from the interacting partners are pooled for the global function of the holobiont [6].

\footnotetext{
* Correspondence: patrick.mavingui@univ-lyon1.fr;

claire.valiente-moro@univ-lyon1.fr

UMR CNRS 5557, USC INRA 1364, VetAgro Sup, Ecologie Microbienne, FR41

BioEnvironment and Health, Université de Lyon 1, Villeurbanne F-69622, France
}

(c) 2013 Minard et al.; licensee BioMed Central Ltd. This is an Open Access article distributed under the terms of the Creative Commons Attribution License (http://creativecommons.org/licenses/by/2.0), which permits unrestricted use, distribution, and reproduction in any medium, provided the original work is properly cited.
There are numerous examples of microbes influencing larly insects which establish as ociation with microbia communities ranging from parasitism to mutualism $[7,8]$. Bacterial endosymbionts are now known to play roles in many key insect functions such as nutrition, reproduction, development or protection against enemies [8]. For example, the facultative bacterium Hamiltonella defensa makes phytophagous aphids more resistant to parasitic wasps [9], whereas the primary symbiont Buchnera aphidicola provides essential amino acids [10]. The bacterium Wigglesworthia glossinidi is thought to provide vitamins to the hematophagous tsetse fly, an important vector of African trypanosomiasis or sleeping sickness, and benefits in return from carbon sources and protection from the insect host [11,12].

Mosquitoes, the Culicidae family, number more than 3,500 different species with a worldwide distribution [13]. Most species described are in the genera Aedes, Anopheles and Culex including several blood feeding members able to transmit pathogens to humans and animals, a great concern for public health [14]. Anopheles mostly transmit parasites such as Plasmodium, whereas Aedes and Culex are responsible for the transmission of arboviruses including Dengue (Flavivirus), Chikungunya (Alphavirus) or Japanese Encephalitis viruses, and filariases such as 
Wuchereria bancrofti or Onchocerca volvulus. Despite intensive efforts, many mosquito-borne diseases (MBD) are increasing worldwide, partly due to the lack of effective vaccines against etiological agents, but also due to global changes in human activities, especially international travel and trade, that have expanded the distribution of mosquito species previously confined to particular regions. To face these outbreaks, new control strategies based on manipulation of the mosquito hosts and their microbial partners have been proposed recently [15]. A well-known example of this in action is the use of the endosymbiotic bacterium Wolbachia [16,17]. This bacterium has a direct impact on the development of some mosquito species by shortening the insect life span, and has indirect effects interfering with pathogen replication and dissemination that affect the vector transmission ability $[18,19]$.

Other than Wolbachia, the interactions between mosquitoes and their associated microbiota have yet to be investigated in depth. Most of the published studies describe bacterial diversity and how it varies according to particular factors. Nevertheless, a common conclusion is that a more comprehensive analysis of symbiotic mosquito interactions is needed at evolutionary and functional levels. Better knowledge of the biological impacts will enable the development of efficient biocontrol approaches for MBD. The present work provides an overview of the diversity of symbiotic bacteria and potential functions in the biology of mosquitoes, and highlights the current and future applications in symbiont-based mosquito control strategies.

\section{Review}

\section{I-Bacterial diversity and variation in mosquitoes}

Complementary approaches are needed for in-depth analyses of microbial communities in complex ecosystems. Both culture-dependent and culture-independent techniques have been used to explore mosquito microbiota. Some microflora can be cultured by using various isolation procedures and media so that bacterial taxa can be identified [20-31] (for details see Additional file 1). The main difficulty of the culture-dependent approach is in recreating the complex physicochemical environment of the insect body [32]. To overcome this limitation and more thoroughly identify bacteria hosted by mosquito populations, culture-independent methods such as Denaturating Gradient Gel Electrophoresis fingerprints, taxonomic microarrays, and meta-taxogenomics can be used (Additional file 1). For example, such molecular approaches, mainly based on analyzing the sequence of the $16 \mathrm{~S}$ ribosomal RNA gene ( $r r s)$, have repeatedly shown the dominance of phylum Proteobacteria in mosquitoes [22-24,28,33-35]. Some bacterial taxa are often underrepresented in results of these global methods, but primers targeting a particular region of rrs or other house- keeping genes can be designed to specifically test for their prevalence in mosquitoes $[28,36,37]$. While these methods are partially successful, they do not give complete overviews of the mosquito-associated bacterial populations. High-throughput sequencing methods are now being implemented to reveal the previously underestimated microbial diversity, and how certain factors impact the composition and structure of these bacterial populations during the life cycle of mosquitoes [33-35]. Microbial communities may be influenced by host intrinsic factors (species, developmental stage, tissue tropism and genetics), the dynamics of intra- and inter-specific interactions and environmental factors.

\section{Host species}

Each mosquito genus has its own preferred habitat and ecological preference. Mosquitoes exhibit particular rhythmic behavioral patterns during their life cycle. For instance, the majority of Anopheles and Culex species are nighttime biters, whereas some Aedes also bite in the daytime. Anopheles mostly live in clear water exposed to sunlight whereas Culex and Aedes are mostly found in dark or troubled water containing a lot of organic matter [38]. In Culicoides midges within the same infra order of Culicidae, it has been demonstrated that the host species could explain $17 \%$ of the variability observed among their bacterial diversity [39]. Surprisingly, there has been no exhaustive comparative study of bacterial diversity across different mosquito species. However, it is possible to compare some surveys and compile the information to highlight specific associations. For instance, when the bacterial content of field populations of adult females of Anopheles stephensi, Anopheles gambiae, Aedes aegypti, Aedes triseriatus and Culex quinquefasciatus was screened with comparable molecular techniques, it emerged that Proteobacteria was the dominant phylum (Figure 1); notably Gammaproteobacteria class representing $41 \%$ (for Cx. quinquefasciatus) to $86 \%$ (for An. stephensi) of the total sequences analyzed, while An. gambiae hosted mainly Alphaproteobacteria and Betaproteobacteria classes, possibly because the mosquito specimens were collected at the larval stage and emerged under laboratory conditions $[22,24,25,34]$. Differences in the proportions of Firmicutes were observed as they account for $13 \%$ of sequences analyzed in Cx. quinquefasciatus, only $1 \%$ in An. stephensi and were not detected at all in Ae. aegypti. Despite these variations, the core bacterial genome present in mosquitoes seems to be similar in different species. Some genera such as Pantoea, Acinetobacter or Asaia are very prevalent in mosquitoes and capable of cross-colonizing different species $[30,40,41]$.

\section{Localization in insect host}

Bacteria colonize different organs in mosquitoes, mainly the midgut and to a lesser extent salivary glands and 


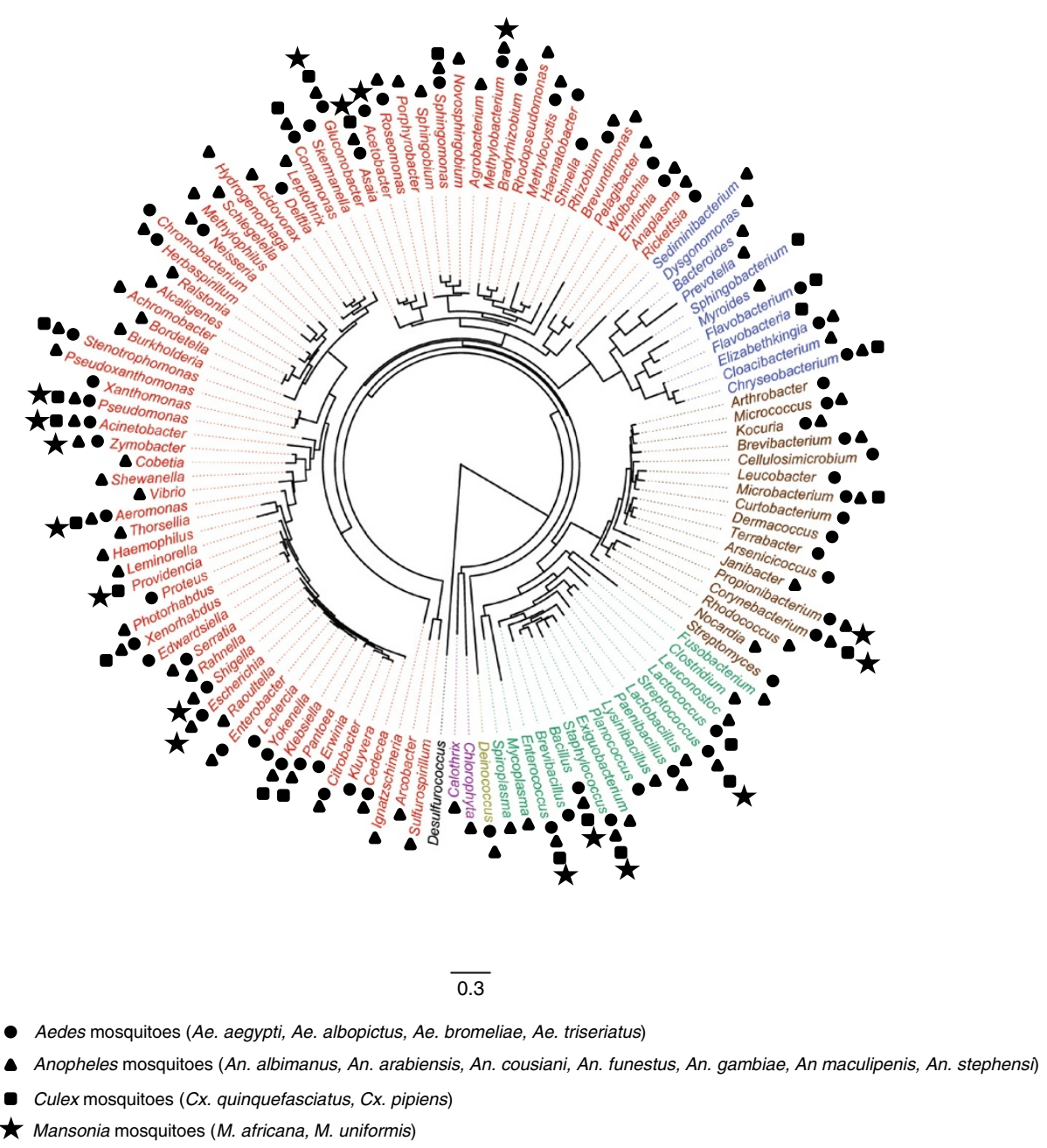

Figure 1 Bacterial genera identified in Culicidae. Bacteria were classified according to their phyla based on branching in the 16S rRNA sequence phylogenetic tree with names shown in color as follows: Proteobacteria (red), Bacteroidetes (blue), Actinobacteria (brown), [Firmicutes, Tenericutes and Fusobacteria] (green), Cyanobacteria (purple) and Deinococcus-Thermus (yellow). The maximum-likelihood tree was built with an HKY model using 100 bootstraps. A 16S rRNA sequence from Desulfurococcus (Archaebacteria) was used as the tree outgroup.

reproductive organs $[22,24,25,28,42,43]$. As in most animal models, the insect gut is a key organ for nutrition and is now considered as being immune-competent $[24,32]$. The gut is an interface with the external environment and provides resources and space that may be favorable to the multiplication of microorganisms ingested [32]. Active gut bacteria contribute to mosquito digestion through the release of lytic enzymes [44]. In some insect species such as aphids, beetles or cockroaches, specialized structures have evolved for microbial endosymbiosis called bacteriocytes or mycetocytes, which are known to be involved in functions including nutrition and immunity $[10,45,46]$. None of these structures has been described in mosquitoes. Insect salivary glands, ovaries and hemolymph are also known to be key organs for virus or parasite replication, but surprisingly the bacterial content of these organs in mosquitoes has not been fully characterized. Nevertheless, these organs were specifically screened for some bacterial endosymbionts. For example, the bacterium Asaia was detected in salivary glands and reproductive systems of different mosquitoes including Ae. aegypti, An. gambiae and An. stephensi $[36,40,47]$. The endosymbiont Wolbachia was also detected in the head, muscles, Malpighian tubules, ovaries and testes of Culex pipiens and Aedes albopictus [48,49]. Strikingly, Wolbachia was also found in Ae. albopictus hemolymph, a fluid which is generally assumed to be bacteria-free [48]. If multiple cell tropisms occur for bacterial partners that are almost fixed in the host population, it is not unreasonable to envisage that a physiological role is yet to be discovered.

\section{Sex of mosquito}

The sex of the mosquito is also an important factor that affects bacterial microbiota composition. Male and female 
mosquitoes exhibit different ecological behaviors in terms of nutritional and dispersal capabilities. Both sexes feed on nectar and plant saps and are able to hydrolyze sucrose, but females are also hematophagous. Indeed, female mosquitoes are anautogenous as they require blood for the completion of their reproductive cycles [50]. In the mosquito digestion process, different hydrolases are released into the anterior and posterior midgut, which constitutes a selective pressure for resident bacteria [51]. Consequently, the composition and distribution of ingested nutrients themselves may also be a constraint for bacterial communities. For instance, a high concentration of carbohydrates and an acidic $\mathrm{pH}$ (from 5.2 to 6.5) occurring in the diverticulum structure are selective for certain bacterial taxa [52,53]. Blood digestion in females is also favored by the selection of bacteria for their hemolytic ability $[25,44]$. Moreover, after a mosquito ingests a blood meal a temperature burst occurs and oxidative stress and immune responses are down regulated, which leads to an increase in the bacterial load $[43,54,55]$.

As mosquito-associated bacteria rely on some of the nutrients brought in the insect meal for growth, the nutrient composition of food sources may directly impact the diversity of bacteria present [24,33]. Zouache et al. (2011) showed that around half of the bacterial diversity in field populations of Ae. albopictus was explained by the sex of the mosquito with greater diversity observed in females [28]. The effect of the sex of the mosquito on bacterial diversity was also reported in field populations of the malaria vector An. stephensi; bacteria from genera Bacillus and Staphylococcus were detected in males, whereas bacteria from genera Cryseobacterium, Pseudomonas and Serratia were present exclusively in females [24]. Considering all published data on mosquito-associated bacteria, it appears that the midgut of females is mostly colonized by members of the Gammaproteobacteria, as is found in other bloodfeeding insects. Interestingly, the genera Pseudomonas, Serratia and Enterobacter are frequently associated with females of several mosquito species [20-24,26,27,29,30,53]. In contrast, the midgut of males is dominated by bacteria from the phylum Firmicutes including those from Staphylococcus, Bacillus, Paenibacillus and Micrococcus genera (Figure 2) [24]. Finally, it was also shown that diet, whether sugar or blood meals, significantly affects the bacterial population structure. Wang et al. (2011) demonstrated that blood meals drastically reduced the community diversity in
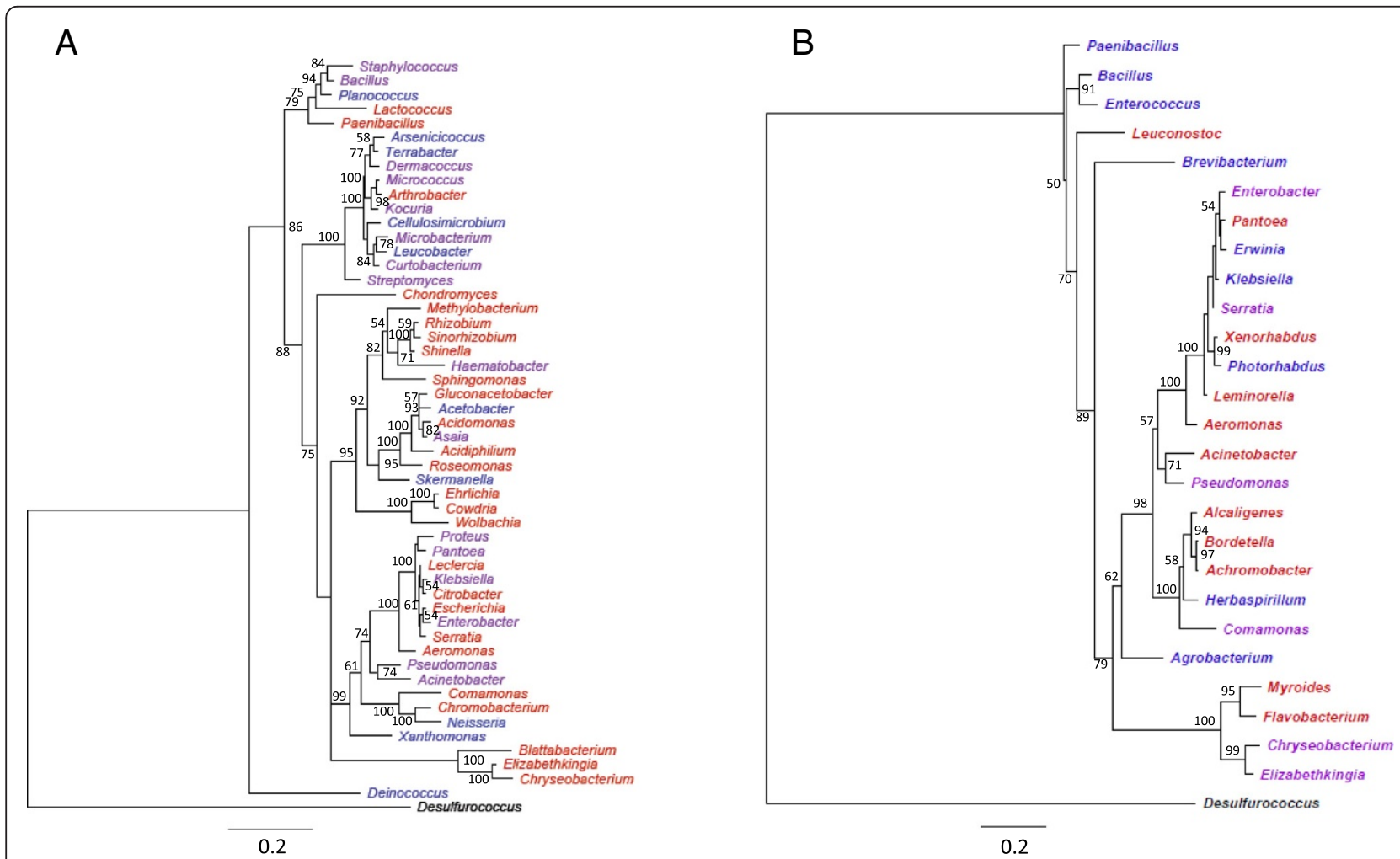

Figure 2 Phylogenetic dendrograms of bacteria identified in mosquito adults. Bacterial genera are classified according to mosquito sex of Ae. albopictus (A) and An. stephensi (B). Names of bacteria identified only in males (blue), only in females (red) or in both males and females (purple) are given. The tree was constructed using the maximum likelihood method with HKY model using 100 bootstraps. Bootstrap values (60\% or above) are shown at branch points. Desulfurococcus (Archaebacteria) was used as the outgroup. 
favor of enteric bacteria in the An. gambiae midgut, while few changes were observed following sugar meals [33]. However, irrespective of the type of meal after 4 days the bacterial microflora reestablishes itself being dominated by the genus Elizabethkingia. Finally, male mosquitoes disperse less than females and tend to remain close to breeding sites which could be an additional factor constraining bacterial diversity [50].

\section{Stages of mosquito development}

Mosquitoes are holometabola that undergo four gradual stages of metamorphosis - egg, larvae, nymph, and adult - that are intimately connected to their respective biotopes. Eggs, larvae and nymphs are aquatic, whereas adult mosquitoes live in terrestrial environments. The fraction of mosquito-associated microflora that is acquired from the surrounding environment is thus likely to differ during the insect life cycle. At the larval stage, individuals consume bacteria and plankton as nutritive resources. This allows a first stage of bacterial colonization that adds to any inherited bacterial flora. Some of these bacteria such as members of the genus Wolbachia are vertically acquired transovarially in $C x$. pipiens, $C x$. quinquefasciatus or Ae. albopictus. Venereal transmission of the bacterium Asaia was reported in An. gambiae and An. stephensi $[47,56,57]$. The midgut of mosquito larvae also contains many photosynthetic cyanobacteria acquired from breeding sites which are not found in adults [58,59]. Wang et al. (2011) showed that in the larval and pupal stages, cyanobacteria were very abundant accounting for $40 \%$ of an entire microbial community in An. gambiae [33]. During its metamorphosis, the mosquito anatomy is radically modified. In particular, a first meconial peritrophic matrix or membrane (MPM1) is formed early in the pupal stadium and a second (MPM2) emerged sometimes around the time of adult emergence [60]. A recent study suggests that MPMs contribute to the sterilization of the adult midgut by sequestering microorganisms ingested during the larval stage, which, along with remaining meconial material, are egested after adult emergence $[60,61]$. This phenomenon could explain why the proportions of different bacterial classes or phyla alter drastically between immature and adult stages. For example, it was shown that the number of bacterial operational taxonomic units (OTU) was 3 fold higher in larvae and pupae than in imagos of An. gambiae [33]. To date, comparative studies of bacterial composition between stages have only been done in Anopheles mosquitoes, in which transtadial maintenance of some bacterial genera such as Acinetobacter, Bacillus, Enterobacter, Staphylococcus, Pseudomonas, Chryseobacterium and Serratia sp. has been observed (Figure 3) [24,26,27,33]. Other mosquito genera should be studied in the same way.

\section{Ecology}

Studies of mosquito-associated bacteria often compare the bacterial communities found in field and lab populations. However, results from lab-reared mosquitoes have revealed the limits of such an approach. By cloning and analyzing signature sequences, Rani et al. (2009) demonstrated that the bacterial diversity of midgut microflora in lab-reared An. stephensi was less than in field-caught ones, both for males (15 versus 27 bacterial taxa) and females (7 versus 36 bacterial taxa) [24]. Similarly, in An. gambiae 45 distinct OTU were identified in lab-reared mosquitoes compared to 155 in field-caught ones using a pyrosequencing approach [34]. Another study of $A n$. gambiae also demonstrated that taxa richness in field-caught mosquitoes was higher than in lab-reared ones for any stage and nutritional condition [33]. Bacterial taxa richness in field-caught mosquitoes shows the extent to which bacteria are acquired from the habitat. Environmental factors should be considered as important drivers impacting the load and composition of bacteria in mosquitoes.

As previously discussed, Culicidae usually live in highly contrasting environments where biotic (like competition or the food chain) and abiotic (like temperature or humidity) factors can influence their microbiota [38]. The complexity of such ecosystems partly explains some of the conclusions drawn from the few existing studies of the role of environmental factors in modulating bacterial composition in field populations of mosquitoes [24]. Currently, the proportion of bacterial species acquired from the environment is unknown [32]. Each mosquito species has ecological preferences that could determine its bacterial content. For instance, some of the adult microflora is acquired from water during mosquito emergence [23]. Plant and animal hosts are a major source of bacterial acquisition through feeding so have a direct impact on the bacterial colonization of mosquitoes [33]. In Culicoides sonorensis, biting midges which transmit viruses to animals, the bacterial flora is derived from soil, plant, bovine and ovine sources [39]. The bacterium Acinetobacter was shown to be frequently associated with different mosquito species, including Ae. aegypti, Ae. albopictus, Ae. triseriatus, An. stephensi, Cx. pipiens, Cx. quinquefasciatus and Psorophora columbiae. Interestingly, this bacterium was also found in mosquito larval breeding sites and in various imago food sources such as vertebrates or plants $[20,22,24,28,29,37,62,63]$. In a similar way the genera Asaia and Pantoea, whose natural habitat is the nectar of tropical flowers, were also observed in mosquitoes $[41,64,65]$. Therefore, the environment may strongly affect the composition of mosquito-associated bacteria. We confirmed this recently by showing that Ae. albopictus individuals from urban areas of Madagascar with bush and fruit tree cover differed from those from suburban areas with bamboo cover [28]. We also demonstrated that the 


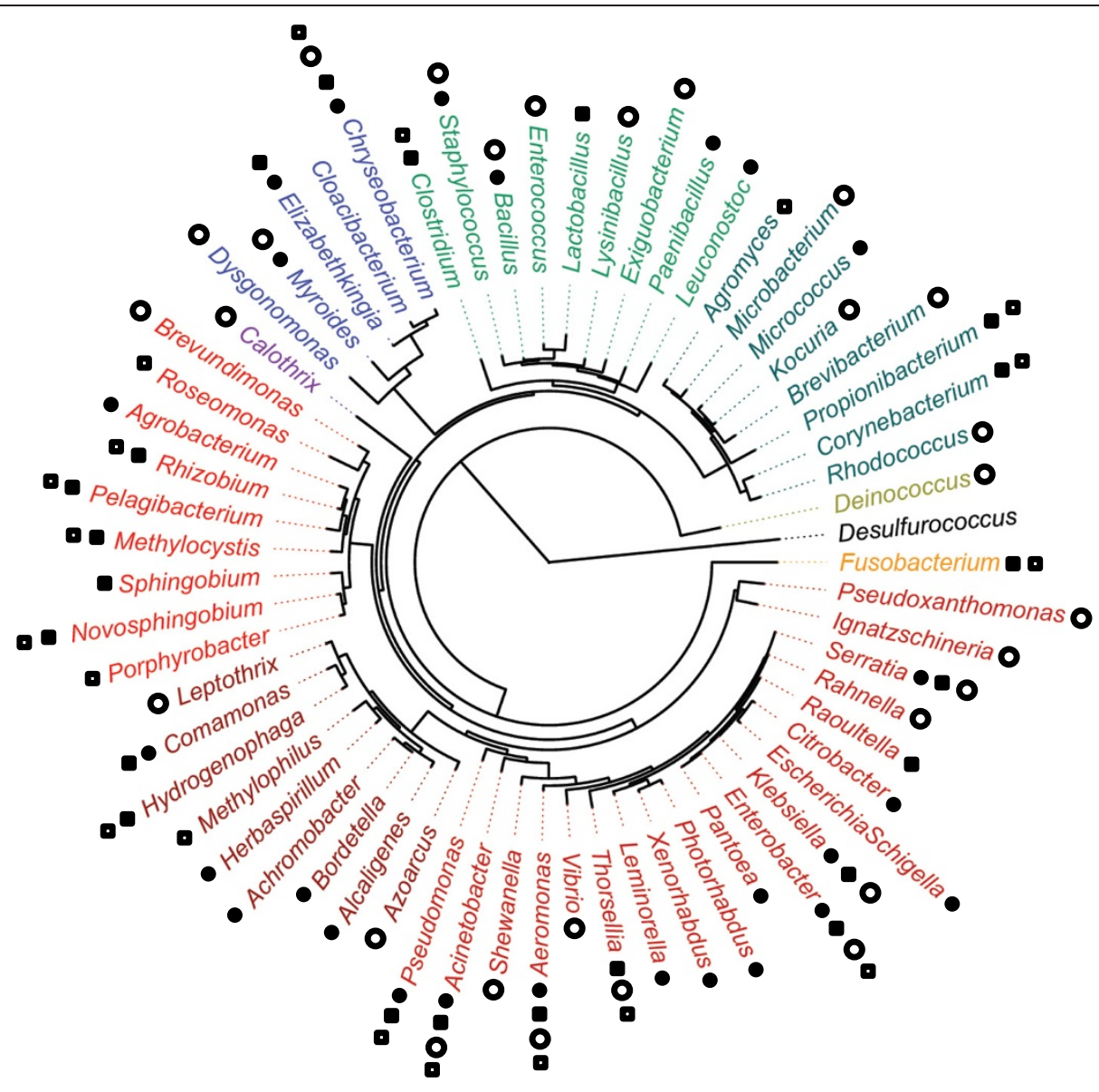

\footnotetext{
- Bacterial genera identified in adult Anopheles stephensi

- Bacterial genera identified in larvae Anopheles stephensi

- Bacterial genera identified in adult Anopheles gambiae

- Bacterial genera identified in larvae Anopheles gambiae
}

Figure 3 Phylogenetic dendrogram of bacterial genera identified in An. stephensi and An. gambiae according to mosquito developmental stage. Names of bacteria are shown in color as follows: Proteobacteria (red), Bacteroidetes (blue), Actinobacteria, Firmicutes, Tenericutes (green), Cyanobacteria (purple), Fusobacteria (orange) and Deinococcus-Thermus (yellow). The tree was constructed using the maximum likelihood method with HKY model using 100 bootstraps. Desulfurococcus (Archaebacteria) was used as the outgroup.

prevalence of Asaia in Ae. albopictus was significantly correlated with the ecological characteristics of sampling sites [37]. Generally, these observations support the idea that field studies are necessary to get an integrated view of mosquito-associated microbiota. However, studies of labreared mosquitoes may be a more convenient alternative to evaluate the impact of abiotic factors on the structure and composition of bacterial communities. For example, Wang and coworkers demonstrated that the main bacterial families Enterobacteriaceae, Flavobacteriaceae and Pseudomonadaceae found in lab-reared An. gambiae were also identified in field-caught individuals from Kenya [33].

\section{Interactions between microbial communities}

Bacterial interactions are important regulators of ecosystem characteristics and species density. These interactions are ranged along the mutualism to parasitism continuum and structure communities [66]. One interesting example is the human gastrointestinal tract. The gut is naturally protected by a heterogeneous bacterial biofilm, a community of microorganisms living inside an adhesive matrix that forms a mutual structure. Pathogen colonization directly alters (dysbiosis) the biofilm structure [67]. Some recent studies focused on the positive and negative interactions between bacteria inside insect 
hosts. Terenius et al. (2012) tested bacterial interspecies competition with isolates from Ae. aegypti and showed that Serratia marcescens could create an inhibition zone area on Sphingomonas and members of the family Burkholderiaceae [30]. The authors suggested a potential link between the presence of $S$. marcescens and the low bacterial diversity observed in the mosquito midgut. Competitive colonization was previously reported in the desert locust Schistocerca gregaria where bacterial diversity was shown to increase in the absence of S. marcescens [68]. Recently, we found a statistically convincing association between the bacteria Asaia and Acinetobacter in Ae. albopictus [37]. Even though additional analyses are still needed to better understand the degree of interactions between the two genera, we showed that bacterial interaction seems to be synergistic because more Asaia-Acinetobacter double-infections were observed than would be expected if the bacteria acted independently.

Bacterial symbionts associated with mosquito vectors have recently been found to interact with pathogens they transmit, modifying the outcome of the multipartite interactions. For instance, it was shown that removing bacterial communities from Anopheles gambiae increased its susceptibility to Plasmodium falciparum infection [69]. On the contrary, Boissière et al. (2012) demonstrated that the presence of some bacteria could favor parasite infection, as they found a positive correlation between the abundance of members of the Enterobacteriaceae family in the mosquito midgut and the Plasmodium infection status [34]. Conversely, Zouache et al. (2012) demonstrated that chikungunya virus infection could modify the diversity of symbiotic bacteria in Ae. albopictus [34,70]. Indeed, taxonomic microarray and quantitative PCR analyses showed that the abundance of Enterobacteriaceae increased with Chikungunya virus infection, whereas the abundance of some other bacterial genera such as Wolbachia and Blattabacterium decreased [70]. All these results suggest that complex microbial interactions (direct or indirect, cooperation or competition) occur between pathogens and microbiota that may affect mosquito traits such as vector competence.

\section{II-Putative impact of bacteria on mosquito biology}

The huge bacterial diversity associated with insects and the complexity of potential interactions between symbiotic microorganisms and their hosts pose a significant challenge to understanding extended phenotypes in mosquitoes. Current technologies are not sufficient to pinpoint all the fluxes of matter and energy between microorganisms and their hosts. However, some beneficial functions provided by bacteria, especially those living intracellularly, the endosymbionts, have been deciphered. Generally, insect-associated bacteria are classified in two broad categories, namely primary and secondary symbionts. Primary symbionts or obligate endosymbionts have co-evolved with their insect hosts while secondary symbionts have become associated with their insect hosts more recently and are not obligate. As yet, there is no description of primary endosymbionts in mosquitoes; all studies focusing on secondary symbionts and their potential role in host biology.

\section{Nutrition}

Bacteria contribute to the nutrition of insects in different ways. Midgut bacteria can produce compounds that are directly assimilated by the host or they can improve digestion by producing degradation enzymes which facilitate the assimilation of complex molecules. In phytophagous insects microbiota generally provide vitamins, amino acids and sterol that complement limited plant diets. The best known example is the involvement of the bacterium Buchnera in providing essential amino acids to aphids [10]. However, a role for bacteria in nutritional complementation in hematophagous insects has not been demonstrated so unequivocally. One interesting example are the bacteria that provide vitamin B which is not present in vertebrate blood, the sole nutrient source of Glossina tsetse flies [71].

In mosquitoes, such a nutritional function has never been formally demonstrated, but some evidence suggests that bacteria could be involved in some processes. For instance, Serratia and Enterobacter, which are known to contain hemolytic enzymes, could play a role in blood digestion in hematophagous Diptera $[25,39,44]$. In Ae. albopictus, Acinetobacter baumannii and Acinetobacter johnsonii could be involved in both blood digestion and nectar assimilation [37]. The evidence for this is that unlike environmental Acinetobacter strains, mosquito isolates were able to metabolize the amino acids $\alpha$-keto -valeric acid and glycine, which are blood components, as well as 4-hydroxy-benzoic acid and xylose, which are common constituents of plant sap. The bacterial species Asaia bogorensis isolated from An. stephensi was shown to be prototrophic with respect to vitamins suggesting it may provide the mosquito with vitamins [40].

Bacteria are involved in nutrition through the release of various compounds useful for mosquito larval development. For instance, it has been demonstrated that a high level of Pseudomonas aeruginosa improved larval growth of $C x$. quinquefasciatus in a phosphorus-rich medium while that of $C x$. tarsalis was slowed down [72]. The level of phosphorus in breeding sites could be a factor explaining how mosquitoes can adapt to a specific condition according to their bacterial load, possibly with a trade-off between the nutritional and toxic roles of bacteria. Differential tolerance of larvae to putative toxins 
present in $P$. aeruginosa could explain why the two mosquito species are not found in the same aquatic habitat.

\section{Reproduction}

As previously shown, some bacteria colonize the reproductive organs of insects allowing them to manipulate host reproduction, allowing them to spread considerably through host populations. The genus Wolbachia is able to control mosquito mating by a phenomenon called cytoplasmic incompatibility. This process prevents infected males from producing viable progeny when mating with an uninfected female or a female infected with an incompatible Wolbachia strain. In this way, certain mosquito species of Aedes and Culex are dependent on Wolbachia to produce viable offspring. Besides Wolbachia, other bacteria could play a role in reproduction, such as the genera Bacillus and Staphylococcus suspected to affect the fertility of the mosquito $C x$. pipiens, although the mechanisms remain to be determined [73].

\section{Other potential functions}

Bacteria occurring in the environment where mosquitoes mature may also impact on their behavior. This is the case for bacteria producing specific odorant compounds that can act as attractants towards mosquitoes. It was demonstrated that the composition of skin microbiota affects the degree of attractiveness of humans to mosquito species [74]. For example, Corynebacterium minutissimum produces volatile compounds such as lactic acid or butyl butyrate that attract An. gambiae [74]. Moreover, bacteria from breeding sites or water-soluble compounds secreted by those bacteria are able to stimulate the hatching of $A e$. aegypti eggs [75]. Some studies demonstrated a link between the presence of bacteria in insect hosts and their ability to degrade some insecticide molecules. For instance, the stinkbug which lives on sugarcane may harbor some fenithrotion-resistant Burkholderia which are acquired from the environment [76]. The acquisition of these bacteria by each generation could be an easy way for the insect to detoxify itself from the insecticide without any genetic cost. As yet, very few studies have described the role of bacteria in the degradation of xenobiotic molecules, though this could be important in understanding why the number of insecticide-resistant mosquitoes is growing. The load of Wolbachia in Cx. pipiens seems to be positively correlated with insecticide resistance mediated by esterase genes at some metabolic cost to mosquitoes [77].

Finally, the effects of experimental depletion of the symbiotic strain Asaia SF2.1 in An. stephensi larvae strongly suggest that the bacterium is a beneficial symbiont of this insect. Indeed, the observation of a delay in the development in larvae after antibiotic treatment in parallel with a dramatic reduction of Asaia burden, led to the hypothesis that this bacterium plays a beneficial role in the development of the mosquitoes [78]. Even though the mechanism remains to be identified, the high prevalence of Asaia combined with their ability to be transmitted both horizontally and vertically provide evidence of the biological role of bacterium in these mosquitoes $[36,47,79]$.

\section{III-Potential applications of bacteria against mosquito vectors} Application of chemical insecticides is still the most common method for mosquito vector control. However, negative consequences like the emergence of insecticideresistant mosquitoes, environmental contamination and damage to non-target organisms have called chemicalbased methods of control into question [80]. The use of bacteria to biologically control mosquito vectors has become a promising strategy.

\section{Cytoplasmic incompatibility}

In the last decade, one of the first efficient strategies to reduce crop insect pests was the introduction of sterile males into a population that, for instance, succeeded in limiting the expansion of the fruit fly Ceratitis capitata [81]. The major difficulty of applying the sterile insect technique (SIT) in mosquito populations was the loss of fitness observed in sterilized males [82-84]. Related to SIT, the incompatible insect technique (IIT) was developed based on Wolbachia-mediated cytoplasmic incompatibility [85]. The trans-infection of Ae. albopictus with Wolbachia strains $w \mathrm{Ri}$ and $w$ Pip Istambul originating from Drosophila simulans and $C x$. pipiens, respectively, caused a significant reduction in hatching rates [86,87]. Interestingly, when $C x$. pipiens was trans-infected with strain $w$ Pip Istambul, no impact was observed on the mosquito's fitness, making this a more promising approach than SIT $[82,83,87]$.

\section{Paratransgenesis}

Transgenesis has also been proposed as a valuable method for controlling mosquito populations. This method is based on the introduction of a transgene in insect vectors which can directly impact on their life history traits or vector competence or indirectly interfere with pathogen replication and transmission [88]. One inconvenience of using transgenic mosquitoes is the cost to mosquito fitness as they are much less competitive [89]. Rather than modifying the insect genome per se, a complementary approach called paratransgenesis was proposed, which consists of using a genetically-modified symbiont known to have an impact on insect life history traits [90,91]. Recently, Asaia was proposed as a promising symbiotic control agent for paratransgenesis as this bacterium is transformable and can be used to express candidate genes in key organs of infected mosquito species. Similarly, Pantoea, a newly identified mosquito symbiont that cross-colonizes several 
mosquito species and can be transformed and cultured, was also proposed for paratransgenic applications [27]. Recently, transgenic strains of Pantoea agglomerans were generated by transformation with a plasmid expressing antiplasmodial compounds [92].

\section{Modification of vector competence}

Vector competence is the ability of a vector to transmit a pathogen, i.e. the intrinsic permissiveness of a vector to be infected, then to replicate and to transmit a pathogen [93]. One strategy used to fight vector-borne pathogens is to decrease vector competence. There is a growing interest in discovering how bacteria interfere with pathogen transmission. In particular, several studies have shown that Wolbachia can decrease or inhibit pathogen replication or transmission in different mosquito species. In general, bacteria successfully interfered with pathogens when mosquitoes were trans-infected with strains isolated from a different host. This is the case for Ae. aegypti and Anopheles which are not naturally infected with Wolbachia. In such artificial systems, a significant reduction in life span and pathogen load (including viruses such as Dengue and Chikungunya or parasites such as plasmodiums and filariases) has been observed $[16,18,19,94,95]$. In Cx. pipiens which is naturally infected by Wolbachia, the West Nile virus load was reduced only 2-3 fold compared to individuals lacking Wolbachia [96]. More recently, the Ae. albopictus ALPROV line naturally harboring two Wolbachia strains, wAlbA and $w \mathrm{AlbB}$, was shown to efficiently replicate the dengue virus but transmission, as measured by the amount of genomic RNA and infectious particles in salivary glands, was significantly reduced compared to the Wolbachia-uninfected line [97]. Mechanisms of bacterial interference of vector competence still remain to be deciphered, but some hypotheses have been suggested. As bacteria and pathogens can invade similar tissues or even the same cells, a theoretical assumption is that they could directly compete for resources and space [98]. The presence of bacteria could also induce the immune system by producing specific compounds that directly interact with pathogens like antiviral or antiparasitic compounds. Recently, Pan et al. (2012) demonstrated that the inhibition of dengue virus in the presence of Wolbachia was correlated with the induction of oxidative stress in the mosquito Ae. aegypti [99]. This response resulted in an activation of the Toll pathway allowing the production of antioxidant molecules and anti-microbial peptides (defensin and cecropins) against dengue virus. In An. gambiae, it was shown that oxidative compounds secreted by the strain Enterobacter Esp_Z. induced a large decrease in Plasmodium in mosquitoes [100]. Joyce et al. (2011) showed that half of the bacterial species isolated from Ae. albopictus midguts decreased the infectivity of the La Crosse virus in animal cells [101].

\section{Bacteria used as insecticides}

Some bacterial strains are able to produce insecticidal compounds that act like natural pesticides. The bacterium Bacillus thuringiensis serovar israelensis produces two different toxins encoded by the cry and cyt genes located on a plasmid replicon [102]. The Cry toxins act on a large insect spectrum (Coleoptera, Lepidoptera, Hymenoptera and Diptera), whereas the Cyt toxins act specifically on Diptera [103]. Both toxins are activated by the alkaline $\mathrm{pH}$ of the larval gut and are able to degrade the midgut membrane causing larvae to die [104]. The Firmicute Lysinibacillus sphaericus also contains the insecticidal Mtx and Bin toxins that are highly active against mosquito larvae [105]. These toxins paralyze the digestive system and disrupt the insect nervous system. These two classes of larvicidal bacteria are major mosquitocidal candidates and were successfully used in the field to reduce An. gambiae populations responsible for malaria outbreaks in Gambia and Ghana [106,107]. Finally, larvicidal toxins of Clostridium bifermentans serovar Malaysia and the pupicidal toxin of Bacillus subtilis subspecies subtilis are also potential candidates as agents in biological control of mosquito populations $[108,109]$.

\section{Conclusions}

Even though information on the nature of mosquitoassociated bacteria is increasing, their functions and genetic potential are still underexplored. This is partly due to the complexity of interactions in terms of bacterial population dynamics influenced by different biotic and abiotic factors. In the near future, the application of next generation sequencing should improve our knowledge of the essential microbial partners and their roles in mosquito biology. Interestingly, the recent development of techniques such as metagenomics, metatranscriptomics, metaproteomics and metabolomics is opening up the possibility of more comprehensive descriptions of molecular foundations and signatures of the relationships between insects and their microbiomes. Such high-throughput analysis will allow a better understanding of the dynamics and function of the mosquito-associated microbiota. It will be possible to explore bacterial communities in an unprecedented way by highlighting metabolically active bacteria and discovering novel bacterial genes that play important roles in chemical and biological processes of the insect host. Moreover, with global changes that have greatly contributed to increase the density and geographic expansion of mosquito populations, questions are now raised about the possible scenarios of emergence or re-emergence of mosquito-borne diseases worldwide. As microbial symbionts of insects often mediate or constrain adaptation to environmental fluctuations, better knowledge of mosquito-associated bacterial communities will be an important aspect of understanding what drives mosquito adaptation. 


\section{Additional file}

\section{Additional file 1: Bacterial genera identified in mosquitoes and} their habitats. An index of bacterial genera identified in mosquitoes and their habitats is presented according to mosquito species, stage, sex, nutrition type, sampling locality, population type and experimental approach (N/A = Not Applicable).

\section{Competing interests}

The authors declare that they have no competing interests.

\section{Authors' contributions}

GM made the alignments, built the phylogenic trees and drafted the manuscript, CVM and PM helped to draft the manuscript. All authors read and approved the final manuscript.

\section{Acknowledgements}

We thank anonymous reviewers for helpful comments. GM was supported by a grant from Academic Research Cluster 1 of Rhône-Alpes Region. This work was partially funded by the grant EC2CO CNRS, and was carried out with the support of CMIRA Coopéra Rhône-Alpes and within the frameworks of GDRIs "Biodiversité et Développement Durable à Madagascar" and "Biodiversity and Infectious Diseases" as well as COST action F0701

'Arthropod Symbioses: from fundamental to pest disease management'.

Received: 19 March 2013 Accepted: 8 May 2013

Published: 20 May 2013

\section{References}

1. Dale C, Moran NA: Molecular interactions between bacterial symbionts and their hosts. Cell 2006, 126:453-465.

2. Lane $\mathrm{N}$ : Energetics and genetics across the prokaryote-eukaryote divide. Biol Direct 2011, 6:35.

3. Reshef L, Koren O, Loya Y, Zilber-Rosenberg I, Rosenberg E: The coral probiotic hypothesis. Environ Microbiol 2006, 8:2068-2073.

4. Guay J-F, Boudreault S, Michaud D, Cloutier C: Impact of environmental stress on aphid clonal resistance to parasitoids: Role of Hamiltonella defensa bacterial symbiosis in association with a new facultative symbiont of the pea aphid. J Insect Physiol 2009, 55:919-926.

5. Rosenberg E, Koren O, Reshef L, Efrony R, Zilber-Rosenberg I: The role of microorganisms in coral health, disease and evolution. Nat Rev Microbiol 2007, 5:355-362.

6. Rosenberg E, Zilber-Rosenberg I: Symbiosis and development: the hologenome concept. Birth Defects Res C Embryo Today 2011, 93:56-66.

7. Toft C, Andersson SGE: Evolutionary microbial genomics: insights into bacterial host adaptation. Nat Rev Genet 2010, 11:465-475.

8. Douglas AE: Lessons from studying insect symbioses. Cell Host Microbe 2011, 10:359-367.

9. Oliver KM, Russell JA, Moran NA, Hunter MS: Facultative bacterial symbionts in aphids confer resistance to parasitic wasps. Proc Natl Acad Sci USA 2003, 100:1803-1807.

10. Douglas AE: Nutritional interactions in insect-microbial symbioses: aphids and their symbiotic bacteria Buchnera. Annu Rev Entomol 1998, 43:17-37.

11. Akman L, Yamashita A, Watanabe H, Oshima K, Shiba T, Hattori M, Aksoy S: Genome sequence of the endocellular obligate symbiont of tsetse flies, Wigglesworthia glossinidia. Nat Genet 2002, 32:402-407.

12. Pais R, Lohs C, Wu Y, Wang J, Aksoy S: The obligate mutualist Wigglesworthia glossinidia influences reproduction, digestion, and immunity processes of its host, the tsetse fly. Appl Environ Microbiol 2008 , 74:5965-5974.

13. Becker N: Mosquitoes and Their Control. New York: Springer; 2003.

14. Cumberland S: Mosquito wars. Bull World Health Organ 2009, 87:167-168.

15. Ricci I, Damiani C, Capone A, Defreece C, Rossi P, Favia G: Mosquito/ microbiota interactions: from complex relationships to biotechnological perspectives. Curr Opin Microbiol 2012, 15:278-284.

16. Iturbe-Ormaetxe I, Walker T, O' Neill SL: Wolbachia and the biological control of mosquito-borne disease. EMBO Rep 2011, 12:508-518.

17. Calvitti M, Moretti R, Skidmore AR, Dobson SL: Wolbachia strain wPip yields a pattern of cytoplasmic incompatibility enhancing a Wolbachia-based suppression strategy against the disease vector Aedes albopictus. Parasites \& Vectors 2012, 5:254

18. McMeniman CJ, Lane RV, Cass BN, Fong AWC, Sidhu M, Wang Y-F, O'Neill SL: Stable introduction of a life-shortening Wolbachia infection into the mosquito Aedes aegypti. Science 2009, 323:141-144.

19. Moreira LA, Iturbe-Ormaetxe I, Jeffery JA, Lu G, Pyke AT, Hedges LM, Rocha BC, Hall-Mendelin S, Day A, Riegler M, Hugo LE, Johnson KN, Kay BH, McGraw EA, van den Hurk AF, Ryan PA, O'Neill SL: A Wolbachia symbiont in Aedes aegypti limits infection with dengue, Chikungunya, and Plasmodium. Cell 2009, 139:1268-1278.

20. Demaio J, Pumpuni CB, Kent M, Beier JC: The midgut bacterial flora of wild Aedes triseriatus, Culex pipiens, and Psorophora columbiae mosquitoes. Am J Trop Med Hyg 1996, 54:219-223.

21. Gonzalez-Ceron L, Santillan F, Rodriguez MH, Mendez D, Hernandez-Avila JE: Bacteria in midguts of field-collected Anopheles albimanus block Plasmodium vivax sporogonic development. J Med Entomol 2003, 40:371-374.

22. Pidiyar VJ, Jangid K, Patole MS, Shouche YS: Studies on cultured and uncultured microbiota of wild Culex quinquefasciatus mosquito midgut based on 16s ribosomal RNA gene analysis. Am J Trop Med Hyg 2004, 70:597-603.

23. Lindh JM, Terenius O, Faye I: $16 \mathrm{~S}$ rRNA gene-based identification of midgut bacteria from field-caught Anopheles gambiae sensu lato and $A$ funestus mosquitoes reveals new species related to known insect symbionts. Appl Environ Microbiol 2005, 71:7217-7223.

24. Rani A, Sharma A, Rajagopal R, Adak T, Bhatnagar RK: Bacterial diversity analysis of larvae and adult midgut microflora using culture-dependent and culture-independent methods in lab-reared and field-collected Anopheles stephensi-an Asian malarial vector. BMC Microbiol 2009, 9:96.

25. Gusmão DS, Santos AV, Marini DC, Bacci M Jr, Berbert-Molina MA, Lemos FJA: Culture-dependent and culture-independent characterization of microorganisms associated with Aedes aegypti (Diptera: Culicidae) (L.) and dynamics of bacterial colonization in the midgut. Acta Trop 2010, 115:275-281.

26. Chavshin AR, Oshaghi MA, Vatandoost $H$, Pourmand MR, Raeisi A, Enayati AA, Mardani N, Ghoorchian S: Identification of bacterial microflora in the midgut of the larvae and adult of wild caught Anopheles stephensi: a step toward finding suitable paratransgenesis candidates. Acta Trop 2012, 121:129-134.

27. Dinparast Djadid N, Jazayeri H, Raz A, Favia G, Ricci I, Zakeri S: Identification of the midgut microbiota of An. stephensi and An. maculipennis for their application as a paratransgenic tool against malaria. PLoS One 2011, 6:e28484

28. Zouache K, Raharimalala FN, Raquin V, Tran-Van V, Raveloson LHR, Ravelonandro P, Mavingui P: Bacterial diversity of field-caught mosquitoes, Aedes albopictus and Aedes aegypti, from different geographic regions of Madagascar. FEMS Microbiol Ecol 2011, 75:377-389.

29. Ramirez JL, Souza-Neto J, Torres Cosme R, Rovira J, Ortiz A, Pascale JM, Dimopoulos G: Reciprocal tripartite interactions between the Aedes aegypti midgut microbiota, innate immune system and dengue virus influences vector competence. PLoS Negl Trop Dis 2012, 6:e1561.

30. Terenius $\mathrm{O}$, Lindh JM, Eriksson-Gonzales K, Bussière L, Laugen AT, Bergquist H, Titanji K, Faye I: Midgut bacterial dynamics in Aedes aegypti. FEMS Microbiol Ecol 2012, 80:556-565.

31. Apte-Deshpande A, Paingankar M, Gokhale MD, Deobagkar DN: Serratia odorifera a midgut inhabitant of Aedes aegypti mosquito enhances its susceptibility to dengue-2 virus. PLoS One 2012, 7:e40401.

32. Dillon RJ, Dillon VM: The gut bacteria of insects: nonpathogenic interactions. Annu Rev Entomol 2004, 49:71-92.

33. Wang Y, Gilbreath TM 3rd, Kukutla P, Yan G, Xu J: Dynamic gut microbiome across life history of the malaria mosquito Anopheles gambiae in Kenya. PLoS One 2011, 6:e24767.

34. Boissière A, Tchioffo MT, Bachar D, Abate L, Marie A, Nsango SE, Shahbazkia HR, Awono-Ambene PH, Levashina EA, Christen R, Morlais I: Midgut microbiota of the malaria mosquito vector Anopheles gambiae and interactions with Plasmodium falciparum infection. PLoS Pathog 2012 8:e1002742.

35. Osei-Poku J, Mbogo CM, Palmer WJ, Jiggins FM: Deep sequencing reveals extensive variation in the gut microbiota of wild mosquitoes from Kenya. Mol Ecol 2012, 21:5138-5150.

36. Favia G, Ricci I, Damiani C, Raddadi N, Crotti E, Marzorati M, Rizzi A, Urso R, Brusetti L, Borin S, Mora D, Scuppa P, Pasqualini L, Clementi E, Genchi M, Corona S, Negri I, Grandi G, Alma A, Kramer L, Esposito F, Bandi C, Sacchi L, 
Daffonchio D: Bacteria of the genus Asaia stably associate with Anopheles stephensi, an Asian malarial mosquito vector. Proc Natl Acad Sci USA 2007, 104:9047-9051.

37. Minard G, Tran FH, Raharimalala FN, Hellard E, Ravelonandro P, Mavingui P, Valiente MC: Prevalence, genomic and metabolic profiles of Acinetobacter and Asaia associated with field-caught Aedes albopictus from Madagascar. FEMS Microbiol Ecol 2013, 83:63-73.

38. Clements AN: The Biology of Mosquitoes: Sensory Reception and Behaviour. Wallingford: CABI Publishing; 1999.

39. Campbell CL, Mummey DL, Schmidtmann ET, Wilson WC: Cultureindependent analysis of midgut microbiota in the arbovirus vector Culicoides sonorensis (Diptera: Ceratopogonidae). J Med Entomol 2004, 41:340-348.

40. Crotti E, Rizzi A, Chouaia B, Ricci I, Favia G, Alma A, Sacchi L, Bourtzis K, Mandrioli M, Cherif A, Bandi C, Daffonchio D: Acetic Acid Bacteria, Newly Emerging Symbionts of Insects. Appl Environ Microbiol 2010, 76:6963-6970.

41. Valiente Moro C, Tran FH, Nantenaina Raharimalala F, Ravelonandro P, Mavingui P: Diversity of culturable bacteria including Pantoea in wild mosquito Aedes albopictus. BMC Microbiol 2013, 13:70.

42. Noden BH, Vaughan JA, Pumpuni CB, Beier JC: Mosquito ingestion of antibodies against mosquito midgut microbiota improves conversion of ookinetes to oocysts for Plasmodium falciparum, but not $P$. yoelii. Parasitol Int 2011, 60:440-446.

43. Oliveira JHM, Gonçalves RLS, Lara FA, Dias FA, Gandara ACP, Menna-Barreto RFS, Edwards MC, Laurindo FRM, Silva-Neto MAC, Sorgine MHF, Oliveira PL: Blood meal-derived heme decreases ROS levels in the midgut of Aedes aegypti and allows proliferation of intestinal microbiota. PLOS Pathog 2011, 7:e1001320.

44. De Gaio AO, Gusmão DS, Santos AV, Berbert-Molina MA, Pimenta PF, Lemos FJ: Contribution of midgut bacteria to blood digestion and egg production in Aedes aegypti (diptera: culicidae) (L.). Parasites Vectors 2011, 4:105.

45. Douglas AE: Mycetocyte symbiosis in insects. Biol Rev Camb Philos Soc 1989, 64:409-434.

46. Baumann P: Biology bacteriocyte-associated endosymbionts of plant sap-sucking insects. Annu Rev Microbiol 2005, 59:155-189.

47. Damiani C, Ricci I, Crotti E, Rossi P, Rizzi A, Scuppa P, Capone A, Ulissi U, Epis S, Genchi M, Sagnon N, Faye I, Kang A, Chouaia B, Whitehorn C, Moussa GW, Mandrioli M, Esposito F, Sacchi L, Bandi C, Daffonchio D, Favia G: Mosquito-bacteria symbiosis: the case of Anopheles gambiae and Asaia. Microb Ecol 2010, 60:644-654

48. Dobson SL, Bourtzis K, Braig HR, Jones BF, Zhou W, Rousset F, O'Neill SL: Wolbachia infections are distributed throughout insect somatic and germ line tissues. Insect Biochem Mol Biol 1999, 29:153-160

49. Zouache K, Voronin D, Tran-Van V, Mousson L, Failloux A-B, Mavingui P: Persistent Wolbachia and cultivable bacteria infection in the reproductive and somatic tissues of the mosquito vector Aedes albopictus. PloS One 2009, 4:e6388.

50. Foster WA: Mosquito Sugar Feeding and Reproductive Energetics. Ann Rev Entomol 1995, 40:443-474.

51. Nation JL: Insect Physiology and Biochemistry. Boca Raton, Florida: CRC Press: 2002.

52. Gelperin A: Regulation of Feeding. Ann Rev Entomol 1971, 16:365-378

53. Gusmão DS, Santos AV, Marini DC, De Russo ES, Peixoto AMD, Bacci Júnior M, Berbert-Molina MA, Lemos FJA: First isolation of microorganisms from the gut diverticulum of Aedes aegypti (Diptera: Culicidae): new perspectives for an insect-bacteria association. Mem Inst Oswaldo Cruz 2007, 102:919-924

54. Kumar S, Molina-Cruz A, Gupta L, Rodrigues J, Barillas-Mury C: A peroxidase/dual oxidase system modulates midgut epithelial immunity in Anopheles gambiae. Science 2010, 327:1644-1648.

55. Benoit JB, Lopez-Martinez G, Patrick KR, Phillips ZP, Krause TB, Denlinger DL: Drinking a hot blood meal elicits a protective heat shock response in mosquitoes. Proc Natl Acad Sci USA 2011, 108:8026-8029.

56. Kittayapong P, Baisley KJ, Sharpe RG, Baimai V, O'Neill SL: Materna transmission efficiency of Wolbachia superinfections in Aedes albopictus populations in Thailand. Am J Trop Med Hyg 2002, 66:103-107.

57. Cook PE, McGraw EA: Wolbachia pipientis: an expanding bag of tricks to explore for disease control. Trends Parasitol 2010, 26:373-375.

58. Thiery I, Nicolas L, Rippka R, De Tandeau MN: Selection of cyanobacteria isolated from mosquito breeding sites as a potential food source for mosquito larvae. Appl Environ Microbiol 1991, 57:1354-1359.
59. Vázquez-Martínez MG, Rodríguez MH, Arredondo-Jiménez Jl, MéndezSanchez JD, Bond-Compeán JG, Cold-Morgan M: Cyanobacteria associated with Anopheles albimanus (Diptera: Culicidae) larval habitats in southern Mexico. J Med Entomol 2002, 39:825-832.

60. Moncayo AC, Lerdthusnee K, Leon R, Robich RM, Romoser WS: Meconial peritrophic matrix structure, formation, and meconial degeneration in mosquito pupae/pharate adults: histological and ultrastructural aspects. J Med Entomol 2005, 42:939-944.

61. Moll RM, Romoser WS, Modrzakowski MC, Moncayo AC, Lerdthusnee K: Meconial peritrophic membranes and the fate of midgut bacteria during mosquito (Diptera: Culicidae) metamorphosis. J Med Entomol 2001, 38:29-32.

62. Jung JY, Park MS, Kim SE, Park BH, Son JY, Kim EY, Lim JE, Lee SK, Lee SH, Lee KJ, Kang YA, Kim SK, Chang J, Kim YS: Risk factors for multi-drug resistant Acinetobacter baumannii bacteremia in patients with colonization in the intensive care unit. BMC Infect Dis 2010, 10:228

63. Shi Y, Lou K, Li C: Growth and photosynthetic efficiency promotion of sugar beet (Beta vulgaris L.) by endophytic bacteria. Photosynthesis Res 2010, 105:5-13.

64. Lindh JM, Borg-Karlson A-K, Faye I: Transstadial and horizontal transfer of bacteria within a colony of Anopheles gambiae (Diptera: Culicidae) and oviposition response to bacteria-containing water. Acta Trop 2008, 107:242-250.

65. Chouaia B, Rossi P, Montagna M, Ricci I, Crotti E, Damiani C, Epis S, Faye I, Sagnon N, Alma A, Favia G, Daffonchio D, Bandi C: Molecular evidence for multiple infections as revealed by typing of Asaia bacterial symbionts of four mosquito species. Appl Environ Microbiol 2010, 76:7444-7450.

66. Moënne-Loccoz Y, Mavingui P, Combes C, Normand P, Steinberg C: Microorganismes et interactions biotiques. In Ecologie microbienne: Microbiologie des milieux naturels et anthropisés. Edited by Bertrand J-C, Caumette P, Lebaron P, Matheron R, Normand P. Publications de I'Université de Pau et des Pays de l'Adour; 2011:413-470.

67. Reid G, Younes JA, Van der Mei HC, Gloor GB, Knight R, Busscher HJ: Microbiota restoration: natural and supplemented recovery of human microbial communities. Nat Rev Microbiol 2011, 9:27-38.

68. Dillon R, Charnley K: Mutualism between the desert locust Schistocerca gregaria and its gut microbiota. Res Microbiol 2002, 153:503-509.

69. Dong Y, Manfredini F, Dimopoulos G: Implication of the mosquito midgut microbiota in the defense against malaria parasites. PLOS Pathog 2009, 5:e1000423

70. Zouache K, Michelland RJ, Failloux A-B, Grundmann GL, Mavingui P: Chikungunya virus impacts the diversity of symbiotic bacteria in mosquito vector. Mol Ecol 2012, 21:2297-2309.

71. Aksoy S: Tsetse-A haven for microorganisms. Parasitol Today (Regul Ed) 2000, 16:114-118.

72. Peck GW, Walton WE: Effect of bacterial quality and density on growth and whole body stoichiometry of Culex quinquefasciatus and Culex tarsalis (Diptera: Culicidae). J Med Entomol 2006, 43:25-33.

73. Fouda MA, Hassan MI, Al-Daly AG, Hammad KM: Effect of midgut bacteria of Culex pipiens L. on digestion and reproduction. J Egypt Soc Parasitol 2001, 31:767-780.

74. Verhulst NO, Andriessen R, Groenhagen U, Bukovinszkiné Kiss G, Schulz S, Takken W, Van Loon JJA, Schraa G, Smallegange RC: Differential attraction of malaria mosquitoes to volatile blends produced by human skin bacteria. PLoS One 2010, 5:e15829.

75. Ponnusamy L, Böröczky K, Wesson DM, Schal C, Apperson CS: Bacteria stimulate hatching of yellow fever mosquito eggs. PLoS One 2011, 6:e24409.

76. Kikuchi Y, Hosokawa T, Fukatsu T: An ancient but promiscuous hostsymbiont association between Burkholderia gut symbionts and their heteropteran hosts. ISME J 2011, 5:446-460

77. Berticat C, Rousset F, Raymond M, Berthomieu A, Weill M: High Wolbachia density in insecticide-resistant mosquitoes. Proc Biol Sci 2002, 269:1413-1416.

78. Chouaia B, Rossi P, Epis S, Mosca M, Ricci I, Damiani C, Ulissi U, Crotti E, Daffonchio D, Bandi C, Favia G: Delayed larval development in Anopheles mosquitoes deprived of Asaia bacterial symbionts. BMC Microbiol 2012, 12(Suppl 1):S2.

79. Damiani C, Ricci I, Crotti E, Rossi P, Rizzi A, Scuppa P, Esposito F, Bandi C, Daffonchio D, Favia G: Paternal transmission of symbiotic bacteria in malaria vectors. Curr Biol 2008, 18:R1087-R1088.

80. David J-P, Coissac E, Melodelima C, Poupardin R, Riaz MA, Chandor-Proust A Reynaud S: Transcriptome response to pollutants and insecticides in the 
dengue vector Aedes aegypti using next-generation sequencing technology. BMC Genomics 2010, 11:216.

81. Robinson AS: Genetic sexing strains in medfly, Ceratitis capitata, sterile insect technique programmes. Genetica 2002, 116:5-13.

82. Alphey L: Re-engineering the sterile insect technique. Insect Biochem Mol Biol 2002, 32:1243-1247.

83. Benedict $M Q$, Robinson $A S$ : The first releases of transgenic mosquitoes: an argument for the sterile insect technique. Trends Parasitol 2003, 19:349-355.

84. Munhenga G, Brooke BD, Chirwa TF, Hunt RH, Coetzee M, Govender D, Koekemoer LL: Evaluating the potential of the sterile insect technique for malaria control: relative fitness and mating compatibility between laboratory colonized and a wild population of Anopheles arabiensis from the Kruger National Park, South Africa. Parasites Vectors 2011, 4:208.

85. Werren JH, Baldo L, Clark ME: Wolbachia: master manipulators of invertebrate biology. Nat Rev Microbiol 2008, 6:741-751.

86. Fu Y, Gavotte L, Mercer DR, Dobson SL: Artificial triple Wolbachia infection in Aedes albopictus yields a new pattern of unidirectional cytoplasmic incompatibility. App/ Environ Microbiol 2010, 76:5887-5891.

87. Atyame CM, Duron O, Tortosa P, Pasteur N, Fort P, Weill M: Multiple Wolbachia determinants control the evolution of cytoplasmic incompatibilities in Culex pipiens mosquito populations. Mol Ecol 2011, 20:286-298.

88. Ruang-Areerate T, Kittayapong P: Wolbachia transinfection in Aedes aegypti: a potential gene driver of dengue vectors. Proc Natl Acad Sci USA 2006, 103:12534-12539.

89. Marrelli MT, Moreira CK, Kelly D, Alphey L, Jacobs-Lorena M: Mosquito transgenesis: what is the fitness cost? Trends Parasitol 2006, 22:197-202.

90. Coutinho-Abreu IV, Zhu KY, Ramalho-Ortigao M: Transgenesis and paratransgenesis to control insect-borne diseases: current status and future challenges. Parasitol Int 2010, 59:1-8

91. Hurwitz I, Hillesland H, Fieck A, Das P, Durvasula R: The paratransgenic sand fly: A platform for control of Leishmania transmission. Parasites Vectors 2011, 4:82.

92. Bisi DC, Lampe DJ: Secretion of anti-Plasmodium effector proteins from a natural Pantoea agglomerans isolate by using PelB and HlyA secretion signals. Appl Environ Microbiol 2011, 77:4669-4675.

93. Lambrechts L, Scott TW: Mode of transmission and the evolution of arbovirus virulence in mosquito vectors. Proc Biol Sci 2009, 276:1369-1378.

94. Kambris Z, Blagborough AM, Pinto SB, Blagrove MSC, Godfray HCJ, Sinden RE, Sinkins SP: Wolbachia stimulates immune gene expression and inhibits plasmodium development in Anopheles gambiae. PLoS Pathog 2010, 6:e1001143.

95. Walker T, Moreira LA: Can Wolbachia be used to control malaria? Mem Inst Oswaldo Cruz 2011, 106(Suppl 1):212-217.

96. Glaser RL, Meola MA: The native Wolbachia endosymbionts of Drosophila melanogaster and Culex quinquefasciatus increase host resistance to West Nile virus infection. PLoS One 2010, 5:e11977.

97. Mousson L, Zouache K, Arias-Goeta C, Raquin V, Mavingui P, Failloux A-B: The Native Wolbachia Symbionts Limit Transmission of Dengue Virus in Aedes albopictus. PLoS Negl Trop Dis 2012, 6:e1989.

98. Mideo N: Parasite adaptations to within-host competition. Trends Parasitol 2009, 25:261-268.

99. Pan X, Zhou G, Wu J, Bian G, Lu P, Raikhel AS, Xi Z: Wolbachia induces reactive oxygen species (ROS)-dependent activation of the Toll pathway to control dengue virus in the mosquito Aedes aegypti. Proc Natl Acad Sci USA 2012, 109:E23-E31.

100. Cirimotich CM, Ramirez JL, Dimopoulos G: Native microbiota shape insect vector competence for human pathogens. Cell Host Microbe 2011, 10:307-310

101. Joyce JD, Nogueira JR, Bales AA, Pittman KE, Anderson JR: Interactions between La Crosse virus and bacteria isolated from the digestive tract of Aedes albopictus (Diptera: Culicidae). J Med Entomol 2011, 48:389-394.

102. González JM Jr, Brown BJ, Carlton BC: Transfer of Bacillus thuringiensis plasmids coding for delta-endotoxin among strains of $B$. thuringiensis and B. cereus. Proc Natl Acad Sci USA 1982, 79:6951-6955.

103. Schnepf E, Crickmore N, Van Rie J, Lereclus D, Baum J, Feitelson J, Zeigle DR, Dean DH: Bacillus Thuringiensis and Its Pesticidal Crystal Proteins. Microbiol Mol Biol Rev 1998, 62:775-806.

104. Bravo A, Gill SS, Soberón M: Mode of action of Bacillus thuringiensis Cry and Cyt toxins and their potential for insect control. Toxicon 2007, 49:423-435.
105. Berry C: The bacterium, Lysinibacillus sphaericus, as an insect pathogen. J Invertebr Pathol 2012, 109:1-10.

106. Majambere S, Lindsay SW, Green C, Kandeh B, Fillinger U: Microbial larvicides for malaria control in The Gambia. Malar J 2007, 6:76.

107. Nartey R, Owusu-Dabo E, Kruppa T, Baffour-Awuah S, Annan A, Oppong S, Becker N, Obiri-Danso K: Use of Bacillus thuringiensis var israelensis as a viable option in an Integrated Malaria Vector Control Programme in the Kumasi Metropolis, Ghana. Parasites Vectors 2013, 6:116.

108. Yiallouros M, Storch V, Thiery I, Becker N: Efficacy of Clostridium bifermentans serovar Malaysia on target and nontarget organisms. J Am Mosa Control Assoc 1994, 10:51-55.

109. Geetha I, Manonmani AM, Paily KP: Identification and characterization of a mosquito pupicidal metabolite of a Bacillus subtilis subsp. subtilis strain. Appl Microbiol Biotechnol 2010, 86:1737-1744.

doi:10.1186/1756-3305-6-146

Cite this article as: Minard et al:: Diversity and function of bacterial microbiota in the mosquito holobiont. Parasites \& Vectors 2013 6:146.

\section{Submit your next manuscript to BioMed Central and take full advantage of:}

- Convenient online submission

- Thorough peer review

- No space constraints or color figure charges

- Immediate publication on acceptance

- Inclusion in PubMed, CAS, Scopus and Google Scholar

- Research which is freely available for redistribution

Submit your manuscript at www.biomedcentral.com/submit
C) Biomed Central 